\title{
The EL2 trap in highly doped GaAs:Te
}

\author{
A. Castaldini, A. Cavallini, ${ }^{\text {a) }}$ and B. Fraboni \\ Dipartimento di Fisica, Universita' di Bologna, via Irnerio 4640126 Bologna, Italy \\ J. Piqueras \\ Departamento de Fisica de Materiales, Universidad Complutense de Madrid, E-28040 Madrid, Spain
}

(Received 21 December 1994; accepted for publication 18 July 1995)

\begin{abstract}
We have investigated highly doped GaAs:Te at different doping concentrations $\left(>10^{17} \mathrm{~cm}^{-3}\right)$ to assess the presence of the EL2 trap. We have utilized both capacitance and current transient spectroscopy techniques. The crucial parameter for the detection of EL2 is the relative position of the electron quasi-Fermi level in the depletion region. The observed shift of the EL2 apparent activation energy with increasing doping concentration is also discussed. () 1995 American Institute of Physics.
\end{abstract}

\section{INTRODUCTION}

The EL2 electron trap is considered the dominant deep level in melt-grown GaAs and it is generally attributed to a complex involving the antisite defect $\mathrm{As}_{\mathrm{Ga}}$, even though the actual structure of the defect is still controversial. One of the most useful techniques for the investigation of deep traps is deep level transient spectroscopy (DLTS) which is based on the analysis of capacitance transients generated by pulsed electronic excitation of a depletion region. It has been observed in the literature that in DLTS measurements the EL2 trap concentration decreases with increasing doping concentration up to a complete disappearance of the trap peak for dopant concentrations $>10^{17} \mathrm{~cm}^{-3}$ Ref. 1 . To account for this effect, it has been proposed that the defect complex related to the EL2 level interacts with the shallow donors and undergoes a stoichiometric transformation as the free-carrier concentration increases, annihilating for concentrations above $10^{17} \mathrm{~cm}^{-3}$ Ref. 2. Another explanation relies on the large reverse saturation current values measured in Schottky diodes fabricated on highly doped material, ${ }^{3}$ which induce variations in the trap occupancy factor. Alternatively, alterations in the Schottky barrier height on highly doped material are considered responsible for the DLTS measurements results. ${ }^{4}$ Capacitance methods have, however, been criticized in the past for their limited capabilities in characterizing highly doped semiconductors, both because of the high electric field in the depletion region and because of the large reverse saturation current of the diode. ${ }^{1}$

This article deals with capacitance (DLTS) and current transient spectroscopy (CTS) and photo-induced current transient spectroscopy (PICTS) investigations on samples with different doping concentrations, in order to explore the actual existence of the EL2 trap in highly doped GaAs. To this aim we have utilized several spectroscopic techniques, as they exploit different physical parameters for the detection of the trap levels. We have indeed observed the EL2 level disappear in the DLTS spectra, but not in the CTS and PICTS ones. This disappearance can be explaincd by taking into account the relative position of the EL2 and quasi-Fermi level in the band gap.

a)Electronic mail: cavallini@bologna.infn.it

\section{EXPERIMENT}

We have studied three sets of LEC GaAs:Te samples with different doping concentrations: set A with $N_{D^{-}} N_{A}=2 \times 10^{17} \mathrm{~cm}^{-3}$, set B with $N_{D^{-}} N_{A}=4 \times 10^{17} \mathrm{~cm}^{-3}$ and set $\mathrm{C}$ with $N_{D^{-}} N_{A}=7 \times 10^{17} \mathrm{~cm}^{-3}$. Schottky diodes have been prepared by evaporating a 500 - $\AA$-thick Au barrier and an In ohmic contact. From $I-V$ measurements the diode ideality factor $n$ has been determined to vary from $n=1.0$ to $n=1.1$ with increasing dopant concentration. The Schottky diodes series resistance resulted always $\leqslant 80 \Omega$. The barrier height $\Phi_{b}$ and the free carrier concentration $N_{D}-N_{A}$ have been obtained from $C-V$ measurements. Table I reports $\Phi_{b}$ and the diode reverse saturation current $J_{s}$ for the three sets of samples at $T=300 \mathrm{~K}$ and a reverse bias $V_{b}=-1 \mathrm{~V}$.

Junction spectroscopy experiments have been carried out with a SULA Tech. system by measuring capacitance transients for DLTS and current transients for CTS and PICTS measurements. The following experimental conditions have been utilized for DLTS and CTS measurements: for set A $V_{b}=-3 \mathrm{~V}$ and a filling pulse $V_{f}=0 \mathrm{~V}$, for sets $\mathrm{B}$ and $\mathrm{C}$ $V_{b}=-0.5 \mathrm{~V}$ and $V_{f}=+1 \mathrm{~V}$. The pulse width has been maintained constant and equal to $100 \mu \mathrm{s}$, while the emission rate $e_{n}$ has been varied from 4.65 to $465 \mathrm{~s}^{-1}$. In PICTS measurements the optical excitation, generated by a LED with $\lambda=670 \mathrm{~nm}$, consisted of a $30 \mathrm{~ms}$ pulse over a $500 \mathrm{~ms}$ period. The reverse bias applied to the diodes was the same as for DLTS measurements. In samples B and C the strong band bending localized in the very narrow depletion region imposed the use of a positive filling pulse. 5

\section{RESULTS}

The Schottky diodes prepared on samples from the three different sets showed significant differences. As can be inferred from Fig. 1, which shows the variation of the reverse saturation current with temperature, the slope of the curve is larger in samples A while the current varies less with temperature in samples $B$ and $C$. The markedly different behavior of samples A with respect to $B$ and $C$ is also verified in the junction spectroscopy results reported below.

Figure 2 shows DLTS, CTS, and PICTS spectra typical of samples A where two peaks are present: the EL2 peak with a level $E_{t}$ located $0.80 \mathrm{eV}$ from the conduction band 
TABLE I. Schottky diode barrier heights, $\Phi_{b}$, and reverse saturation currents, $J_{s}$, at $T=300 \mathrm{~K}$ for the three sets of samples investigated.

\begin{tabular}{ccc}
\hline Set & $\Phi_{b}(\mathrm{eV})$ & $J_{s}\left(\mathrm{~A} / \mathrm{cm}^{2}\right)$ \\
\hline $\mathrm{A}$ & 0.75 & $1.2 \times 10^{-3}$ \\
$\mathrm{~B}$ & 0.71 & $1.3 \times 10^{-2}$ \\
$\mathrm{C}$ & 0.69 & $5.0 \times 10^{-2}$ \\
\hline
\end{tabular}

$E_{c}{ }^{6}$ and another peak at lower temperature with $E_{\mathrm{c}}-E_{t}=0.34 \mathrm{eV}$. Their capture cross sections are $\sigma_{n}=4 \times 10^{-13}$ and $\sigma_{n}=4.3 \times 10^{-15} \mathrm{~cm}^{2}$, respectively. A trap similar to the latter one has already been detected in LEC GaAs:Te and named ECX. ${ }^{7}$ The EL2 peak position is slightly shifted in the DLTS spectrum with respect to the CTS and PICTS ones, as can be expected for the differences existing between current and capacitance transient techniques. In fact, it has been calculated that, for a given rate window, the emission rate $e_{n}$ relative to a current transient is larger than for a capacitance transient. This accounts for the different position of the same peak in the temperature axis. ${ }^{8}$ The trap concentration has been determined taking into account the diode series resistance ${ }^{9}$ and the so-called " $\lambda$-effect", 10,11 and they resulted to be $[E L 2]=1.6 \times 10^{16}$ $\mathrm{cm}^{-3}$ and $[\mathrm{ECX}]=2.4 \times 10^{14} \mathrm{~cm}^{-3}$.

The spectra taken from samples of set $\mathrm{C}$ are quite different, as can be inferred from Fig. 3 where DLTS, CTS, and PICTS results are reported. A shift of the ECX level has been

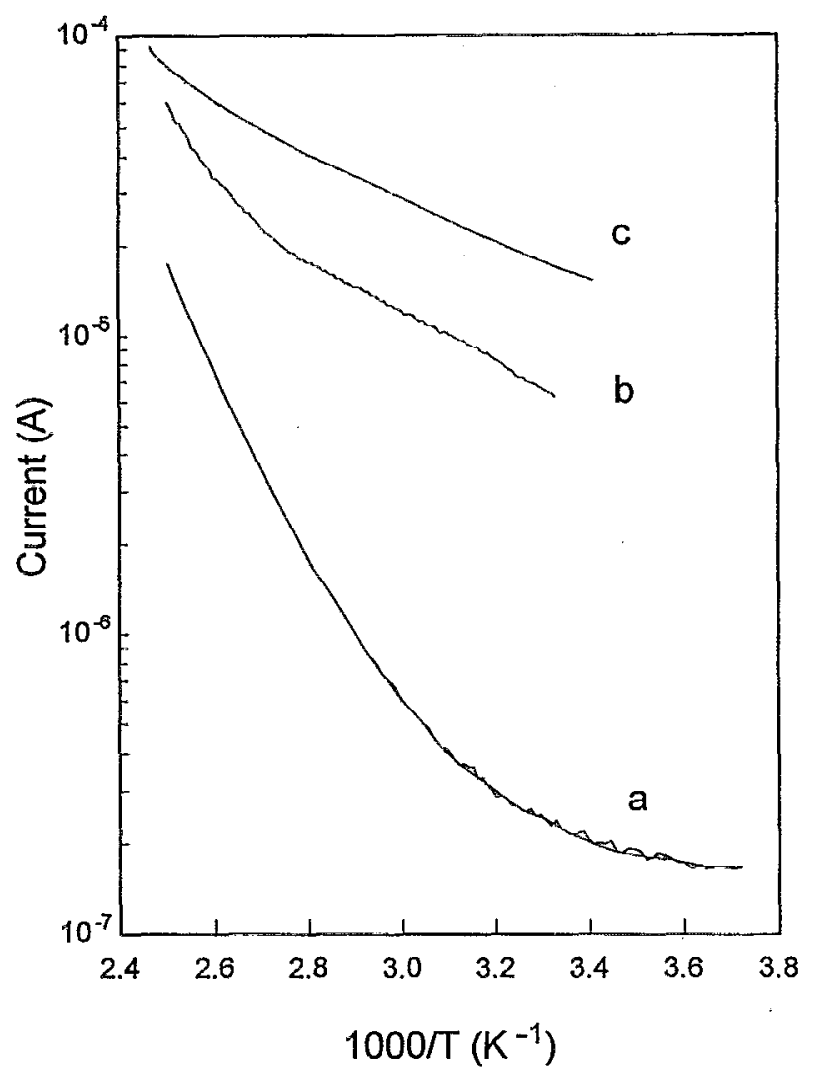

FIG. 1. Schottky diode reverse current as a function of temperature at $V_{b}=-1 \mathrm{~V}$, for samples of set A (curve $a$ ), B (curve $b$ ), and C (curve $c$ ).

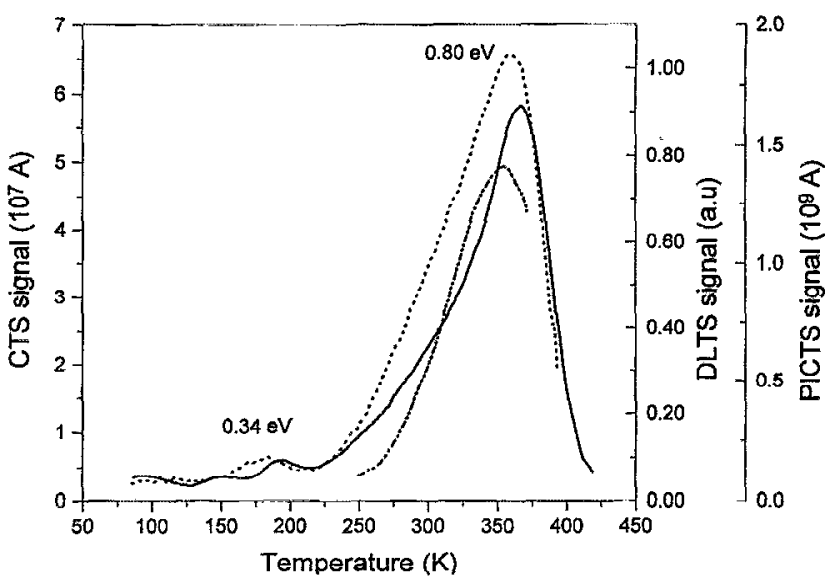

FIG. 2. DLTS, CTS, and PICTS spectra typical of samples from set A. The solid line refers to the DLTS, the dotted line to the CTS and the chain line to the PICTS signal. The following experimental conditions have been used: $V_{b}=-3 \mathrm{~V}, V_{f}=0 \mathrm{~V} e_{n}=46.5 \mathrm{~s}^{-1}$.

observed, from 0.34 to $0.30 \mathrm{eV}$. Focusing the attention on the EL2 peak, whose activation energy shifts to $0.76 \mathrm{eV}$, it is evident that it is not present in DLTS, while it is still visible in CTS and PICTS spectra. The detection of other peaks (ECX and a level at $E_{c}-E_{t}=0.59 \mathrm{eV}$ with $\sigma_{n}=9 \times 10^{-12}$ $\left.\mathrm{cm}^{2}\right)$ in DLTS, indicates that the disappearance of EL2 is not only due to the poor capabilities of capacitance spectroscopy methods, as proposed by Hasegawa et $a .^{3}$ Rather, these findings suggest that it is due to the relative position in the gap of EL2 and of the quasi-Fermi level (QFL). We have calculated the QFL position for each set of samples, according to the procedure of $\mathrm{Ma}$ et al., ${ }^{4}$ taking into account the measured EL2 energy level for each sample (EL2 $2_{A}=0.80 \mathrm{eV}$, $\mathrm{EL} 2_{B}=0.78 \mathrm{eV}$, and $\left.\mathrm{EL} 2_{C}=0.76 \mathrm{eV}\right)$. We have neglected the contribution to the $I-V$ characteristic of the tunneling and image-force effects, as it has been demonstrated that they do not seriously affect the results of the QFL calculations. ${ }^{12}$ The results, relative to the temperature $T=300 \mathrm{~K}$, are shown in

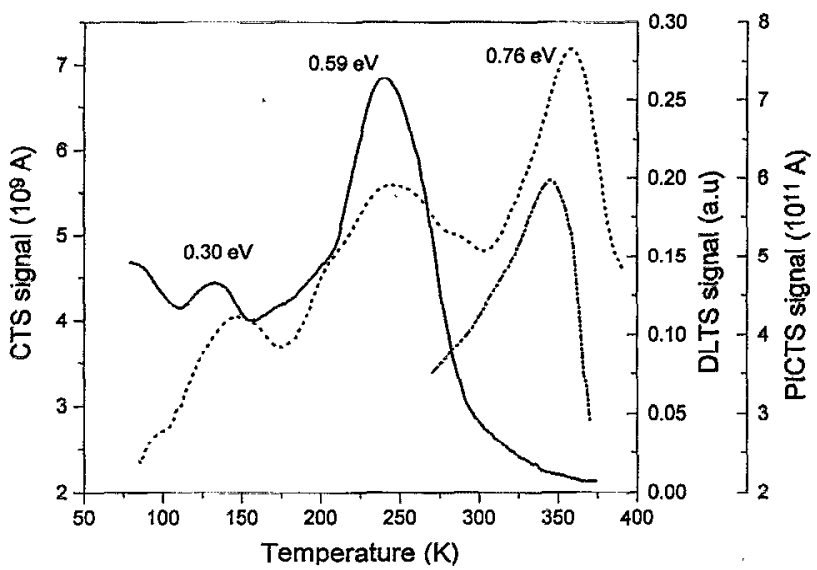

FIG. 3. DLTS, CI'S, and PICTS spectra typical of samples from set C. The solid line refers to the DLTS, the dotted line to the CTS and the chain line to the PICTS signal. The following experimental conditions have been used: $V_{b}=0 \mathrm{~V}, V_{f}=+1 \mathrm{~V}, e_{n}=46.5 \mathrm{~s}^{-1}$. 


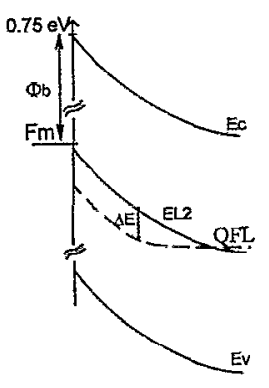

a

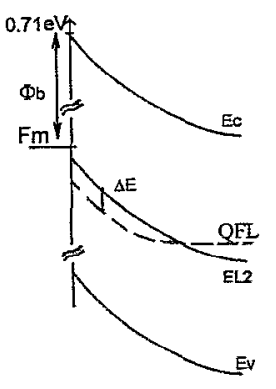

b

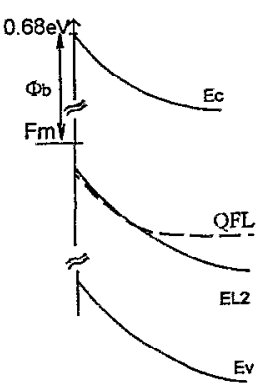

c
FIG. 4. Band diagram of the depletion region relevant to the metalsemiconductor contact for the three sets of samples, at $T=300 \mathrm{~K}$. Figures 4(a)-4(c) refer, respectively, to sets $\mathrm{A}, \mathrm{B}$, and C. $F_{m}$ is the metal Fermi level, $\Phi_{b}$ is the barrier height, QFL is the quasi-Fermi level. The distance $\Delta E$ between EL2 and QFL is $\Delta E=0.09 \mathrm{eV}, \Delta E=0.05 \mathrm{eV}$, and $\Delta E=0.02$ $\mathrm{eV}$ for sets $\mathrm{A}, \mathrm{B}$, and $\mathrm{C}$, respectively.

Fig. 4. Figure 5 shows the computed $\Delta E=E L 2-Q F L$ variation with temperature for sets $\mathrm{A}, \mathrm{B}$, and $\mathrm{C}$, obtained considering the reverse saturation current trend (Fig. 1). The determination of the trap concentrations proved to be difficult for samples $\mathrm{B}$ and $\mathrm{C}$, since the experimental conditions utilized do not allow to apply the usual data treatment for such an evaluation.

The Arrhenius plots obtained for EL2 in the three sets are reported in Fig. 6, where squares refer to set $\mathrm{A}$, circles to set $B$ and triangles to set $C$ samples. It is evident that the EL2 Arrhenius plot position shifts along the temperature axis with increasing doping concentration, the resulting activation energy varying from 0.80 to $0.76 \mathrm{eV}$.

\section{DISCUSSION}

The fundamental assumption for the detection of a deep electron trap is that its energy level lies above the QFL. For this reason we have, first of all, calculated the relative position of EL2 and QFL in our sets of samples, utilizing the

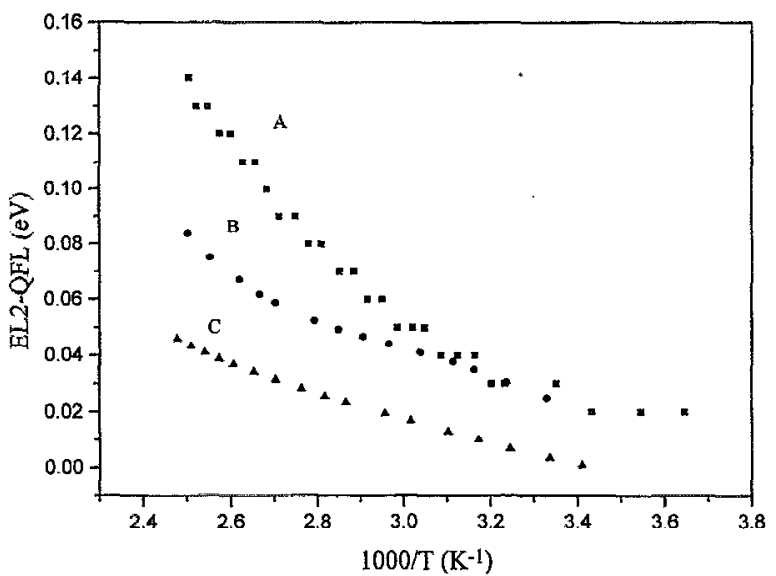

FIG. 5. Variation with temperature of the separation in the band gap between the EL2 and the quasi-Fermi level for samples A (curve $a$ ), B (curve $b$ ), and $\mathbf{C}$ (curve $c$ )

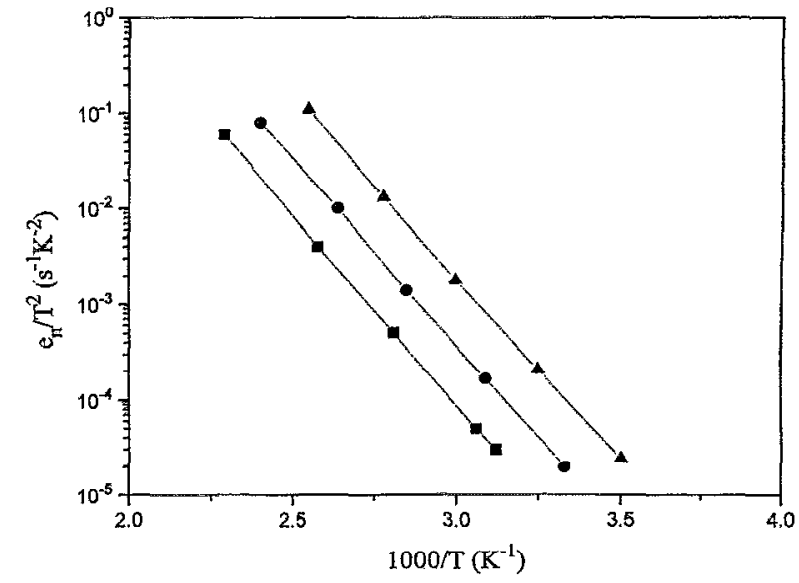

FIG. 6. Arrhenius plots of the EL2 level in samples A (squares), B (circles), and $\mathrm{C}$ (triangles).

reverse saturation current trend with temperature. Even though we have observed that the separation $\Delta E$ between the two levels decreases by increasing the doping density, the EL2 lcvel maintains above QFL for every experimental condition applied and for every sample (Fig. 4). This indicates that the appearance of the EL2 peak is strongly related to the physical process utilized to identify the electron traps, i.e., to the experimental technique employed.

The variation of the reverse saturation current with temperature (Fig. 1) confirms the trend calculated for $\Delta E$ (Fig. 5). It is evident that the deterioration of the diode performances with increasing doping density is related to the separation between EL2 and QFL. In samples A and B $\Delta E>k T$ in the whole temperature range investigated, while in samples $C$ for $T \geqslant 360 \mathrm{~K}$, the temperature when the EL2 peak starts to appear, $\Delta E \sim \mathrm{kT}(\sim 0.026 \mathrm{eV})$. The EL2 occupancy actually changes during the filling pulse in all of the investigated samples. This variation is expectedly very small in the most doped samples and, thus, hardly appreciable if the detectivity limit of the technique employed is not low enough. As the detectivity limit of the DLTS method is at least five times higher than in CTS, ${ }^{13}$ the EL2 peak cannot be detected in the DLTS spectra of the most doped samples while it is still present in CTS. In the other samples the separation between the QFL and the trap level largely exceeds the thermal noise, thus permitting the trap detection. This last consideration holds for every other electron trap taking into account the relevant $\Delta E$.

The increase of the reverse saturation current in highly doped samples, which hinders the EL2 detection in DLTS, can be suitably suppressed in current transient measurements techniques, where the greater sensitivity of the method allows to detect even small variations in the current signal. ${ }^{13}$ The EL2 peak is always present in CTS and PICTS spectra, indicating that such techniques are capable of investigating the high-temperature region of highly doped semiconductors, while DLTS can still be used on such specimens but only in a limited range of temperatures.

The observed shift of the EL2 Arrhenius plot in the three sets of samples could be attributed to different effects in- 
TABLE II. Band gap variation $\Delta E_{g}$ (Ref. 15) estimated for the threc investigated sets of samples. The measured EL2 activation energies are also reported.

\begin{tabular}{ccc}
\hline \hline Set & $\Delta E_{g}(\mathrm{eV})$ & EL2 $\left(E_{\mathrm{c}}-E_{t}\right)(\mathrm{eV})$ \\
\hline $\mathrm{A}\left(2 \times 10^{17} \mathrm{~cm}^{-3}\right)$ & 0.015 & 0.80 \\
$\mathrm{~B}\left(4 \times 10^{17} \mathrm{~cm}^{-3}\right)$ & 0.035 & 0.78 \\
$\mathrm{C}\left(7 \times 10^{17} \mathrm{~cm}^{-3}\right)$ & 0.048 & 0.76 \\
\hline \hline
\end{tabular}

duced by the increased concentration of dopant atoms. In $n$-type semiconductors, the impurity levels in the gap tend to spatially overlap and form a band which merges with the near conduction band, thus adding a "tail" of states. Moreover, the conduction band is perturbed by deformation potentials locally induced by the accommodation of impurities in the lattice, the overall effect being a smearing of the band edge. ${ }^{14}$ The combination of the conduction band filling effect (known as the Burstein shift ${ }^{15}$ ) and of the shrinkage of the effective energy gap, ${ }^{14,16}$ induces a variation $\Delta E_{g}$ of the band gap width. It has been proposed that the band gap shrinkage $\Delta E_{\mathrm{g}}$ could be determined by studying the diode recombina-. tion current as a function of doping concentration and temperature. ${ }^{16}$ We have obtained an estimate of $\Delta E_{g}$ for each set of investigated samples from experimental data taken from the literature. ${ }^{15}$ 'Ihe results are reported in Table II. Such a band gap variation may be considered responsible for the observed shift of the EL2 activation energy with increasing doping concentration of the sample, as the measured $E_{c}-E_{t}$ values are affected by the actual bottom of the conduction band $E_{r}$.

Another explanation of the observed shift of the EL2 activation energy could be a stoichiometric transformation of the defect, accompanied by a more general rearrangement of the material as the dopant concentration increases, hypothesis which is supported by the appearance of a third peak in the most doped samples $\left(E_{c}-E_{t}=0.59 \mathrm{eV}\right){ }^{2,17}$ However, the EL2 trap manifests itself in different ways in LEC GaAs and it is quite impossible to attribute all the experimental data in the literature to a unique origin or defect complex. It has been suggested that a family of midgap electron traps exists in LEC GaAs, with similar Arrhenius plot but different electrical and optical properties. ${ }^{18}$

\section{CONCLUSIONS}

We have studied LEC GaAs:Te with different doping concentration by junction spectroscopy methods (DLTS, CTS, and PICTS). Two peaks have been detected in the less doped set of samples, set $A$, one identified as EL2 $\left(E_{c}-E_{t}=0.80 \mathrm{eV}\right)$ and the other as $\operatorname{ECX}\left(E_{c}-E_{t}=0.34\right.$ $\mathrm{eV})$. In the most doped samples, sets $\mathrm{B}$ and $\mathrm{C}$, a third peak appears at $E_{c}-E_{t}=0.59 \mathrm{eV}$. In the DLTS spectra of the most doped samples the EL2 peak does not appear, while it is still present in the CTS/PICTS ones. This indicates that EL2 does not annihilate in highly doped GaAs:Te. Its disappearance results to be a measurement artefact in the DLTS method, imputable to the thermal noise which becomes comparable to the distance between EL2 and the quasi-Fermi level. This effect strongly affects capacitance spectroscopy methods which result less reliable than current spectroscopy methods in the high-temperature range. Furthermore, the Arrhenius plot of the EL2 level moves towards lower temperatures with increasing doping concentration. This could be explained by the combined effect of changes in the density of states near the conduction band edge (band tails), of dopant induced strain in the lattice, of the Burstein shift for $n$-type materials and of majority carrier screening. ${ }^{14-16}$ In fact, the net result of all the above mentioned effects is the narrowing with increasing doping concentration of the band gap width and a variation of the measured activation energies of deep traps. An alternative explanation of the observed shift could be a rearrangement of the atomic configuration of EL2 induced by the increasing dopant concentration. This last hypothesis is supported by the appearance of a third level in the most doped sets of samples.

\section{ACKNOWLEDGMENTS}

This research has been partially supported by the Cooperation Programme "Azione Integrata" between Italy and Spain.

${ }^{1}$ G. Martin and S. Makram-Ebeid, in Deep Centres in Semiconductors, edited by S. T. Pantelides (NY Gordon and Breach, New York, 1986), Chap. 6 , pp. $399-488$.

${ }^{2}$ J. Lagowski, H. C. Gatos, J. M. Parsey, K. Wada, M. Kaminska, and W. Walukievicz, Appl. Phys. Lett. 40, 342 (1982).

${ }^{3} \mathrm{~F}$. Hasegawa and M. Onomura, in Semi-Insulating III-V Materials, Malmö 1988, edited by G. Grossman and L. Ledebo (A. Hilger, Bristol, 1989), p. 149.

${ }^{4}$ Q. Y. Ma, M. T. Schmidt, X. Wu, H. L. Evans, and E. S. Yang, J. Appl. Phys. 64, 2469 (1988).

${ }^{5}$ A. Cola, M. G. Lupo, L. Vasanelli, and A. Valentini, Solid-State Electron. 36, 785 (1993).

${ }^{6}$ G. M. Martin, A. Mitonneau, and A. Mircea, Electron. Lett. 13, 191 (1977).

${ }^{7}$ D. Wong, H. K. Kim, Z. Q. Fang, T. E. Schlesinger, and A. G. Milnes, J. Appl. Phys: 66, 2002 (1989).

${ }^{8}$ D. Pons, Ph.D. thesis, Universite' de Paris VI (Paris, 1979).

${ }^{9}$ C. Kisielowsky and E. R. Weber, Phys. Rev. B 44, 1600 (1991).

${ }^{10} \mathrm{P}$. Blood and J. W. Orton, The Electrical Characterization of Semiconduc* tors: Majority Carriers and Electron States (Academic, London, 1992), Chap. 7, pp. 336-397; Chap. 8, pp. 399-465.

${ }^{11}$ Y. Zotha and M. O. Watanabe, J. Appl. Phys. 53, 1809 (1982).

${ }^{12}$ C. R. Crowell and M, Beguwala, Solid-State Electron. 14, 1149 (1971).

${ }^{13} \mathrm{~J}$. Borsuk and R. Swanson, IEEE Trans. Electron. Dev. ED-27, 2217 (1980).

14 J. I. Pankove, Phys. Rev. A 140, 2059 (1965).

${ }^{15}$ E. Burstein, Phys. Rev. 93, 632 (1954).

${ }^{16}$ A. Neugroschel, S. C. Pao, and F. A. Lindholm, IEEE Trans. Electron. Dev. ED-29, 894 (1982).

${ }^{17}$ R. Yakimova, T. Paskova, and Ch. Hardalov, J. Appl. Phys. 74, 6170 (1993).

${ }^{18}$ M. Taniguchi and T. Ikoma, J. Appl. Phys. 54, 6448 (1983). 
Journal of Applied Physics is copyrighted by the American Institute of Physics (AIP). Redistribution of journal material is subject to the AIP online journal license and/or AIP copyright. For more information, see http://ojps.aip.org/japo/japcr/jsp

Copyright of Journal of Applied Physics is the property of American Institute of Physics and its content may not be copied or emailed to multiple sites or posted to a listserv without the copyright holder's express written permission. However, users may print, download, or email articles for individual use. 
Journal of Applied Physics is copyrighted by the American Institute of Physics (AIP). Redistribution of journal material is subject to the AIP online journal license and/or AIP copyright. For more information, see http://ojps.aip.org/japo/japcr/jsp 